\title{
Overexpression of Zwint predicts poor prognosis and promotes the proliferation of hepatocellular carcinoma by regulating cell-cycle-related proteins
}

This article was published in the following Dove Press journal: OncoTargets and Therapy

\author{
Hanning Ying ${ }^{1,2}$ \\ Zhiyao $\mathrm{Xu}^{3}$ \\ Mingming Chen ${ }^{1,2}$ \\ Senjun Zhou ${ }^{1,2}$ \\ Xiao Liang ${ }^{1,2}$ \\ Xiujun Cai ${ }^{1,2}$ \\ 'Department of General Surgery, \\ ${ }^{2}$ Key Laboratory of Endoscopic \\ Technique Research of Zhejiang \\ Province, ${ }^{3}$ Central Lab of Biomedical \\ Research Center, School of Medicine, \\ Sir Run Run Shaw Hospital, Zhejiang \\ University, Hangzhou, China
}

Introduction: Zwint, a centromere-complex component required for the mitotic spindle checkpoint, has been reported to be overexpressed in different human cancers, but it has not been studied in human hepatocellular carcinoma (HCC).

Materials and methods: The role of Zwint in hepatocellular carcinoma cell proliferation capacities was evaluated by using cell counting kit-8 (CCK8), flow cytometry, clone formation and tumor formation assay in nude mice. Western blot analysis and qPCR assay were performed to assess Zwint interacting with cell-cycle-related proteins.

Results: We report that ZWINT mRNA and protein expression were upregulated in HCC samples and cell lines. An independent set of 106 HCC-tissue pairs and corresponding noncancerous tissues was evaluated for Zwint expression using immunohistochemistry, and elevated Zwint expression in HCC tissues was significantly correlated with clinicopathological features, such as tumor size and number. Kaplan-Meier survival and Cox regression analysis revealed that high expression of Zwint was correlated with poor overall survival and a greater tendency for tumor recurrence. Ectopic expression of Zwint promoted HCC-cell proliferation, and Zwint expression affected the expression of several cell-cycle proteins, including PCNA, cyclin B1, Cdc25C and CDK1.

Conclusion: Our findings suggest that upregulation of Zwint may contribute to the progression of HCC and may be a prognostic biomarker and potential therapeutic target for treating HCC. Keywords: Zwint, hepatocellular carcinoma, HCC, prognosis, cell proliferation, cell cycle

\section{Introduction}

Hepatocellular carcinoma (HCC) is the fifth-most common malignancy and the third-leading cause of cancer deaths worldwide. ${ }^{1} \mathrm{HCC}$ is an aggressive cancer associated with poor survival, frequent recurrence, and metastases after surgical resection or chemotherapy. ${ }^{2}$ Moreover, most patients are diagnosed with highly invasive, unresectable HCC, and have poor outcomes. Although advanced diagnostics and new treatments have been developed, how HCC progresses is not clear. ${ }^{3}$ As such, there is an urgent need to identify novel biomarkers to improve patient prognosis.

Zwint, a centromere-complex component required for the mitotic spindle checkpoint, is involved in centromere function and cell growth. ${ }^{4}$ Most studies have suggested that Zwint interacts with Zw10, another centromere protein, possibly regulating the association between Zw10 and centromeres. Zwint is reported to be required for spindle-assembly checkpoint function. ${ }^{5}$ In addition, Zwint has been shown to be a part of the MIS12 complex and interacts with Rab3C. ${ }^{6-8}$ Endo et al demonstrated that Zwint depletion by Terf/Trim17 negatively regulated cell proliferation in $293 \mathrm{~T}$ cells and breast cancer MCF7 cells. ${ }^{9}$ Seo et al reported that Zwint was required to
Correspondence: Xiujun Cai Department of General Surgery, Sir Run-Run Shaw Hospital, Zhejiang University, 3 Qingchun East Road, Xin Cheng Shang Quan, Jianggan Qu, Hangzhou, Zhejiang 310016, China Email 0094417@zju.edu.cn hereby accept the Terms. Non-commercial uses of the work are permitted without any furcther permission from Dove Medical Press Limited, provided the work is properly attributed. For permission for commercial use of this work, please see paragraphs 4.2 and 5 of our Terms (https://www.dovepress.com/terms.php). 
correct erroneous centromere-microtubule attachment and regulate spindle-checkpoint function during meiosis. ${ }^{10}$ Recently, overexpression of Zwint has been reported in human malignancies, including prostate, ovarian, bladder, and breast cancers, as well as pulmonary adenocarcinoma and ependymomas. ${ }^{11-16}$ Aberrant expression of Zwint is associated with chromosome-instability signatures and poor prognosis in primary tumors. ${ }^{17}$ These new findings encourage further investigation of Zwint's potential clinical significance in human malignancies.

Because the expression and clinical significance of Zwint has not been studied in human HCC, we performed searches and survival analyses of Zwint on microarray data sets from the Oncomine (www.oncomine.com) and The Cancer Genome Atlas (TCGA) databases. We examined ZWINT mRNA and protein expression in HCC clinical samples and analyzed correlations of Zwint expression with various clinical and pathological parameters. In addition, we explored the role of Zwint in regulating cell proliferation in vivo and in vitro. Our results showed that Zwint increases tumor growth and may be a novel prognostic marker in HCC.

\section{Materials and methods}

\section{Ethics approval and informed consent}

The study was verified and ethically approved by the Medical Ethics Committee of Sir Run Run Shaw Hospital (SRRSH), College of Medicine, Zhejiang University. All patients provided written informed consent for the use of surgical specimens for pathological testing with operation consents. As such, the Medical Ethics Committee of (SRRSH) decided that the study of patient tissue for future research was ethically acceptable without special informed consent. Patient privacy is well protected, and no personal information is disclosed in this article. All animal care and experimental procedures were in strict accordance with recommendations in the Guide for the Care and Use of Laboratory Animals, and were approved by the Committee on the Ethics of Animal Experiments of Zhejiang Chinese Medical University (permit ZSLL-2017-096).

\section{Patients and specimens}

Formalin-fixed paraffin-embedded tissue samples from 106 HCC patients who had received curative surgery in SRRSH from January 2006 to December 2010 were included. Clinical and pathological characteristics of the cohort are listed in Table 1. Tumor stages were graded according to the seventh edition of the American Joint Committee on Cancer staging system. ${ }^{18}$ Tumor differentiation was classified according to the Edmondson-Steiner grading system, and
Table I Association between Zwint expression and clinicopathologic characteristics of HCC patients $(n=106)$

\begin{tabular}{|c|c|c|c|c|}
\hline & \multirow[t]{2}{*}{ Cases } & \multicolumn{2}{|c|}{ Zwint expression } & \multirow[t]{2}{*}{$P$-value* } \\
\hline & & Low (\%) & High (\%) & \\
\hline Total & 106 & $47(44.3)$ & $59(55.7)$ & \\
\hline \multicolumn{2}{|l|}{ Age (years) } & & & 0.199 \\
\hline$<60$ & 49 & $25(53.2)$ & $24(40.7)$ & \\
\hline$\geq 60$ & 57 & $22(46.8)$ & $35(59.3)$ & \\
\hline \multicolumn{2}{|l|}{ Sex } & & & 0.596 \\
\hline Male & 88 & $38(80.8)$ & $50(84.7)$ & \\
\hline Female & 18 & $9(19.2)$ & $9(15.3)$ & \\
\hline \multicolumn{2}{|l|}{ HBsAg } & & & 0.253 \\
\hline Negative & 16 & $5(10.6)$ & $11(18.6)$ & \\
\hline Positive & 90 & $42(89.4)$ & $48(8 I .4)$ & \\
\hline \multicolumn{2}{|l|}{$\operatorname{AFP}(\mathrm{ng} / \mathrm{mL})$} & & & 0.767 \\
\hline$<400$ & 66 & $30(63.8)$ & $36(6 I)$ & \\
\hline$\geq 400$ & 40 & $17(36.2)$ & $23(39)$ & \\
\hline \multicolumn{2}{|l|}{ Liver cirrhosis } & & & 0.582 \\
\hline No & 46 & $19(40.4)$ & $27(45.8)$ & \\
\hline Yes & 60 & $28(59.6)$ & $32(54.2)$ & \\
\hline \multicolumn{2}{|l|}{ Tumor size } & & & $0.025 *$ \\
\hline$\leq 5 \mathrm{~cm}$ & 64 & $34(72.3)$ & $30(50.8)$ & \\
\hline$>5 \mathrm{~cm}$ & 42 & $13(27.6)$ & $29(49.2)$ & \\
\hline \multicolumn{2}{|l|}{ Tumor number } & & & $0.035^{*}$ \\
\hline Single & 93 & 45 (95.7) & $48(81.4)$ & \\
\hline Multiple & 13 & $2(4.3)$ & $11(18.6)$ & \\
\hline \multicolumn{3}{|c|}{ Tumor differentiation } & & 0.134 \\
\hline Good/moderate & 56 & 21 (44.7) & $35(59.3)$ & \\
\hline Poor & 50 & $26(55.3)$ & $24(40.7)$ & \\
\hline \multicolumn{2}{|l|}{ Tumor thrombi } & & & 0.647 \\
\hline No & 92 & $40(85.1)$ & $52(88.1)$ & \\
\hline Yes & 14 & $7(14.9)$ & 7 (II.9) & \\
\hline \multicolumn{2}{|l|}{ TNM stage } & & & 0.507 \\
\hline$I+I I$ & 96 & $44(93.6)$ & $52(88.1)$ & \\
\hline III + IV & 10 & $3(6.4)$ & $7(11.9)$ & \\
\hline \multicolumn{2}{|l|}{ BCLC stage } & & & I \\
\hline A & 96 & 43 (91.5) & $53(89.8)$ & \\
\hline B & 10 & $4(8.5)$ & $6(10.2)$ & \\
\hline
\end{tabular}

Notes: *Pearson chi-square test or Fisher exact test was used for comparison between subgroups. Significant results $(P<0.05)$ are given in bold.

Abbreviations: $\mathrm{HCC}$, hepatocellular carcinoma; $\mathrm{HBsAg}$, hepatitis $\mathrm{B}$ surface antigen; BCLC, Barcelona Clinic Liver Cancer.

clinical stages were defined based on the Barcelona Clinic Liver Cancer (BCLC) staging system. ${ }^{18,19}$ Patients were followed for 6 years.

A set of 37 paired fresh-frozen HCC samples obtained from patients who had received surgery in our hospital from August 2014 to December 2015 were used for quantitative polymerase chain reaction (PCR) analysis. For Western blot, 20 paired fresh-frozen HCC samples and corresponding noncancerous samples were obtained from HCC patients undergoing hepatectomy from January 2015 to June 2015 in SRRSH.

Roessler liver microarray (250 HCC samples and 220 normal livers), Wurmbach liver microarray (35 HCC samples and ten normal livers) and Chen liver microarray (74 HCC samples and 104 normal livers) were collected from Oncomine. 
mRNA expression from $374 \mathrm{HCC}$ tissues was obtained from the publicly available TCGA database, and the survival rate (364 patients with follow-up information) was analyzed by the Gene Expression Profiling Interactive Analysis website (http://gepia.cancer-pku.cn), a network analyzing TCGA data online. ${ }^{28}$

\section{Cell culture}

The human HCC cell lines Huh7, HepG2, HCCLM3, Hep3B and normal hepatic cell line L02 were obtained from the Type Culture Collection of the Chinese Academy of Sciences (Shanghai, China). HCCLM3, Huh7, Hep3B, and L02 cells were cultured in DMEM (Thermo Fisher Scientific, Waltham, MA, USA) with 10\% fetal bovine serum (FBS) (Thermo Fisher Scientific). HepG2 cells were cultured in MEM (Thermo Fisher Scientific) with 10\% FBS (Thermo Fisher Scientific). Cells were maintained at $37^{\circ} \mathrm{C}$ in $5 \% \mathrm{CO}_{2}$.

\section{Plasmid construction and cell infection}

A lentivirus-based Zwint RNA plasmid (shZWINT) and a nonspecific shRNA plasmid (Scrambled) were constructed (GeneChem, Shanghai, China). The ZWINT gene was cloned in pWPI plasmids. pWPI-Zwint and pWPI-vector plasmids were transfected into $293 \mathrm{~T}$ cells with a mixture of pWPI, PAX2 (viral packaging plasmid), and pMD2G (envelope plasmid) at a 4:3:2 ratio using Lipofectamine 2000 (Thermo Fisher Scientific). Lentiviral supernatants were then collected to infect HCC cells. Stable cells were selected and confirmed by quantitative real-time (qRT) PCR and Western blotting.

\section{Quantitative real-time PCR analysis}

Total RNA from tissue samples and cells were extracted using Trizol reagent (Thermo Fisher Scientific) according to the manufacturer's instructions. cDNA was synthesized from $1 \mu \mathrm{g}$ RNA of each sample using an iScript cDNA-synthesis kit (Bio-Rad Laboratories, Hercules, CA, USA). qRT-PCR was performed using a 7500 RT-PCR system (Thermo Fisher Scientific). Zwint primer sequences were: forward 5'-AGGACACTGCTAAGGGTCTCG-3', reverse 5'-GCCTCTACGTGCTCCCTGTA-3'. Cyclin B1 primer sequences were: forward 5'-AACTTTCGCCT GAGCCTATTTT-3', reverse 5'-TTGGTCTGACTGCT TGCTCTT-3'. PCNA primer sequences were: forward $5^{\prime}$-CC TGCTGGGATATTAGCTCCA-3', reverse 5'-CAGCGGT AGGTGTCGAAGC-3'. CDK1 primer sequences were: forward 5'-GGATGTGCTTATGCAGGATTCC-3', reverse 5'-CATGTACTGACCAGGAGGGATAG-3'. GAPDH primer sequences were: forward 5'-ACAACTTT
GGTATCGTGGAAGG-3', reverse 5'-GCCATCACGCCAC AGTTTC-3'. Each sample was tested in triplicate. Data were analyzed using the $\Delta \Delta \mathrm{Ct}$ method.

\section{Western blot}

Cells were harvested and washed twice with cold PBS, then resuspended and lysed in radioimmunoprecipitation-assay buffer ( $1 \%$ NP40, 0.5\% sodium deoxycholate, $0.1 \%$ sodium dodecyl sulfate, $10 \mathrm{ng} / \mathrm{mL}$ phenylmethylsulfonyl fluoride, $0.03 \%$ aprotinin, $1 \mu \mathrm{M}$ sodium orthovanadate) at $4{ }^{\circ} \mathrm{C}$ for 30 minutes. Lysates were centrifuged for 10 minutes at $14,000 \times g$ and supernatants quantified with a Pierce BCA protein-assay kit (Thermo Fisher Scientific). Protein was separated by $10 \%$ sodium dodecyl sulfate polyacrylamide-gel electrophoresis. Samples were transferred to polyvinylidene difluoride membranes (Merck Millipore, Billerica, MA, USA) and incubated overnight at $4^{\circ} \mathrm{C}$ with the primary antibodies Zwint (1:1,000, AP6686c; Abgent, San Diego, CA, USA), Cdc25C (1:1,000, ab32444; Abcam, Cambridge, UK), cyclin B1 (1:1,000, CST 4138), CDK1 (1:1,000, ab133327; Abcam), PCNA (1:1,000, CST 13110S), and GAPDH (1:1,000, G5262; Sigma-Aldrich, St Louis, MO, USA). After incubation with HRP-coupled anti-mouse IgG antibody $\left(1: 2,000\right.$, Beyotime, Haimen, China) at $37^{\circ} \mathrm{C}$ for 2 hours, target proteins on polyvinylidene difluoride membranes were visualized using Clarity Western ECL substrate (Bio-Rad) and captured using a luminescent-image analyzer (FujiFilm, Tokyo, Japan).

\section{Immunohistochemical analysis}

Immunohistochemistry (IHC) was used to evaluate Zwint expression in 106 paired HCC samples and corresponding noncancerous samples. Primary antibodies for Zwint (1:400, AP6686c) were incubated overnight at $4^{\circ} \mathrm{C}$. Normal goat serum was used as a negative control. Expression of Zwint was then measured using a GTVision III detection system (Gene Tech, Shanghai, China). Diaminobenzidine visualization was then performed. Immunostaining was evaluated and scored by two pathologists without prior knowledge of clinicopathological data using a German immunoreactive score. ${ }^{20}$ Intensity was assessed as 0 (negative), 1 (weak), 2 (moderate), and 3 (strong). Scores representing percentage of tumor cells positively stained were $0(<5 \%), 1(5 \%-25 \%), 2(25 \%-50 \%)$, $3(50 \%-75 \%)$, or $4(>75 \%)$. Staining-intensity values and staining extent were multiplied as a final immunoreactive score of Zwint expression with scores of $0,1,2,3,4,6,8,9$, or 12 . A score $\geq 6$ was used to define tumors with high Zwint expression and a score $\leq 4$ indicated low Zwint expression. 


\section{Cell-proliferation assay}

Stable transfected cells $\left(3 \times 10^{3}\right.$ for HCCLM3 and Huh7, $5 \times 10^{3}$ for HepG2) were seeded on 96-well plates with three replicate wells and allowed to incubate for 24 hours. After incubation, cell viability was assessed at $0,24,48,72$ and 96 hours using a cell counting kit-8 (CCK-8) protocol (Dojindo, Kumamoto, Japan). All experiments were performed at least in triplicate on three separate occasions.

\section{Plate colony-formation assay}

For colony-formation assays with monolayer cultures, HCCLM3 cells were seeded at 300 cells/well in six-well plates with Zwint shRNA plasmid and Zwint Scramble plasmid. HepG2 cells were seeded at 600 cells/well in sixwell plates with pWPI-Zwint and pWPI-vector plasmids. After 14 days of culture, cell colonies ( $\geq 50$ cells/colony) were counted after staining with $0.5 \%$ crystal violet.

\section{Cell-cycle analysis}

Cell-cycle distribution was determined by flow cytometry according to the manufacturer's instructions for the cell-cycle staining buffer (MultiSciences CCS01). Cells were detected with a FACSCalibur.

\section{Tumor-formation assay in nude mice}

BALB/c nude mice ( $n=12,4$ weeks of age) were obtained from Shanghai Laboratory Animal Center and housed in the laboratory-animal research center of Zhejiang Medical University. Cultures of HCCLM3 cells for control and $\operatorname{sh} Z$ WINT groups were resuspended with PBS. Mice were randomized and treated with HCCLM $3 / \mathrm{sh} Z$ WINT (6 $\times 10^{6}$, subcutaneous) or control in $150 \mu \mathrm{L}$ of PBS $(\mathrm{n}=6 /$ group). After 10 days, growth of implanted tumors was monitored using Vernier calipers every 5 days:

Tumor volume $\left(\mathrm{cm}^{3}\right)=0.5 \times$ Tumor length $(\mathrm{cm})$ $\times$ Tumor width ${ }^{2}\left(\mathrm{~cm}^{2}\right)$

All mice were sacrificed after 30 days.

\section{Statistical analysis}

Statistical analysis was performed using SPSS 18.0 software (SPSS Inc, Chicago, IL, USA). GraphPad Prism 6 software (San Diego, CA, USA) was used to perform graphical representations. Associations between Zwint and clinical features were analyzed using Pearson's $\chi^{2}$ or Fisher's exact test. Survival data were analyzed with Kaplan-Meier survival curves and log-rank test. Univariate and multivariate Cox proportional-hazard models were used to evaluate the effect of each variable on survival. Correlations of expression of Zwint and PCNA, cyclin B1, Cdc25C, and CDK1 were analyzed using bivariate correlation analysis. Scatterplots with regression lines confirmed correlations using Pearson's correlation coefficient. All results of experiments are expressed as means \pm SD. Statistical analysis of differences between treated and untreated groups was performed with Student's $t$-test, and $P<0.05$ was considered significant.

\section{Results}

\section{Overexpression of Zwint in HCC tissues and cell lines}

Analysis of multiple microarray data sets in the Oncomine and TCGA databases confirmed that ZWINT mRNA was significantly increased in HCC tissues compared to adjacent normal liver tissues in Roessler (Figure 1A), Wurmbach (Figure 1B), and Chen liver data sets (Figure 1C). ZWINT mRNA expression from 37 paired fresh-frozen HCC tissues was significantly increased compared to corresponding noncancerous tissues $(P<0.01$, Figure 1D). Upregulation of ZWINT mRNA occurred in most cases (Figure 1E). Upregulated ZWINT was associated with shortened patient survival ( $P=0.00061$, Figure 1F). Zwint protein was measured in HCC samples and corresponding adjacent normal liver specimens with Western blot or IHC staining. Figure 2 shows that most HCC samples had elevated Zwint protein (Figure 2A and C), and Zwint expression in four HCC cell lines and a normal human L02 liver cell line confirmed that Zwint expression was greater in HCC cell lines (Figure 2B).

\section{Correlation of Zwint expression with HCC clinicopathologic features}

IHC data for Zwint expression in 106 pairs of HCC tissues and adjacent normal tissues were classified as described in the Materials and methods section. Figure $3 \mathrm{~A}$ shows different staining-intensity levels of tissue samples and that there was a statistically significant difference in Zwint expression between HCC and normal tissue ( $P<0.001$, Figure 3B). Fisher's exact or $\chi^{2}$ test was used to analyze correlations between Zwint protein expression and clinicopathologic variables, and high Zwint expression was significantly correlated with tumor size $(P=0.025)$ and tumor number $(P=0.035)$. However, Zwint overexpression was not associated with other features, as described in Table 1.

\section{Prognostic values of Zwint expression for HCC patients}

Kaplan-Meier analysis and log-rank test were used to assess the association between Zwint expression and 106 patients' 

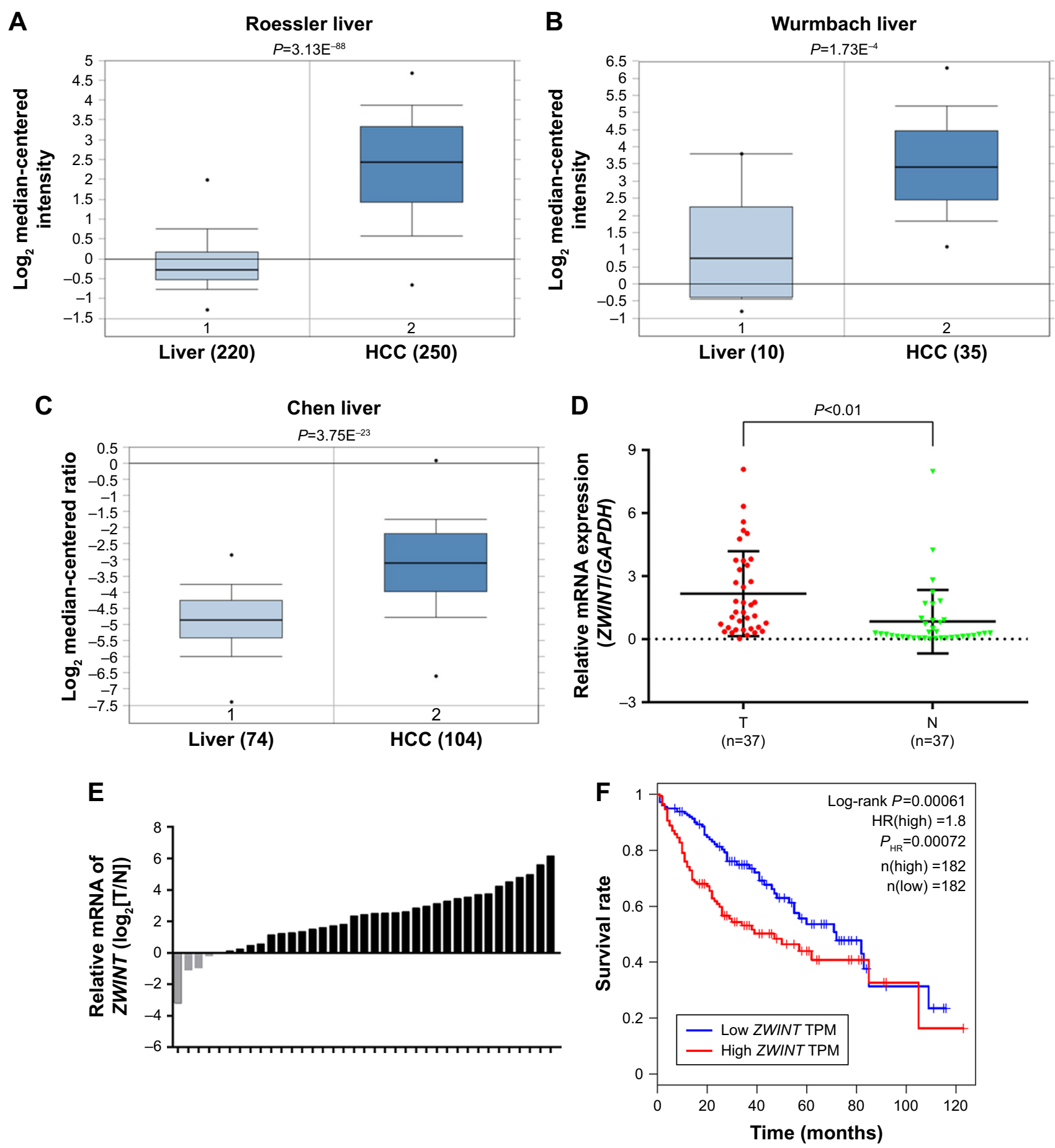

Figure I Expression of ZWINT in HCC at mRNA level.

Notes: (A-C) Upregulated ZWINT mRNA expression compared with normal liver tissue in Roessler, Wurmbach, and Chen liver data sets from the Oncomine database. (D) mRNA level in 37 paired HCC-tissue samples. ZWINT expression was elevated in HCC tissue $(P<0.01)$. (E) ZWINT mRNA levels in 37 paired HCCtissue samples. ZWINT mRNA level expressed as $\log _{2}(T / N)$. $\log _{2}(T / N)<0$ represents the downregulation of $Z W I N T$ in tumor tissue. Log $(T / N)>0$ represents the upregulation of ZWINT in tumor tissue. (F) Based on the TCGA database and analyzed by the Gene Expression Profiling Interactive Analysis website (http://gepia. cancer-pku.cn), a network analyzing TCGA data online, high expression of ZWINT predicted a poor survival rate in 364 HCC patients.

Abbreviations: HCC, hepatocellular carcinoma; T, tumor tissue; N, noncancerous tissue; TCGA, The Cancer Genome Atlas; TPM, transcripts per (kilobase) million; HR, hazard ratio.

prognoses, and Figure 4 shows that 5-year overall and disease-free survival were shorter with high Zwint expression $(P=0.001$, Figure $4 \mathrm{~A})$ and this was also associated with more tumor recurrence $(P=0.005$, Figure $4 \mathrm{~B})$. Median survival time was shorter with high Zwint expression (67 months versus 55 months). Stratified survival analysis to evaluate prognostic significance showed that for tumor size $\leq 5 \mathrm{~cm}$, high expression of Zwint predicted poor overall survival 

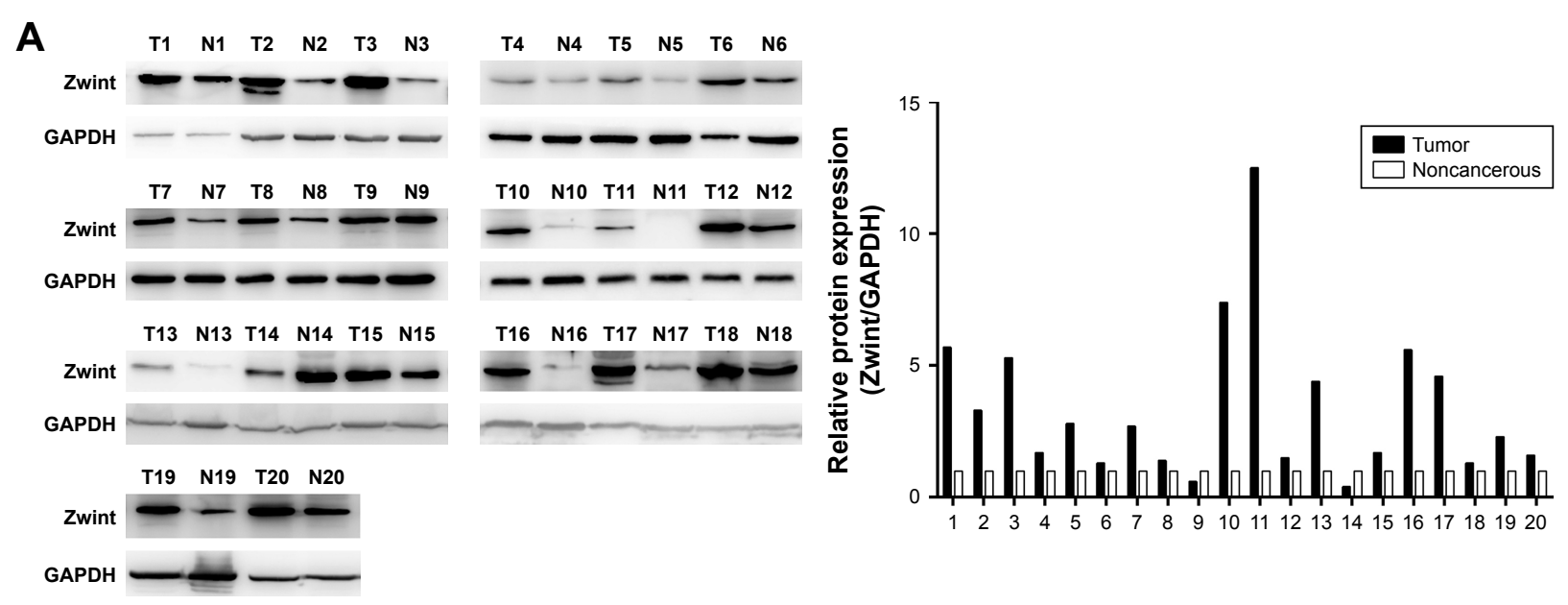

B
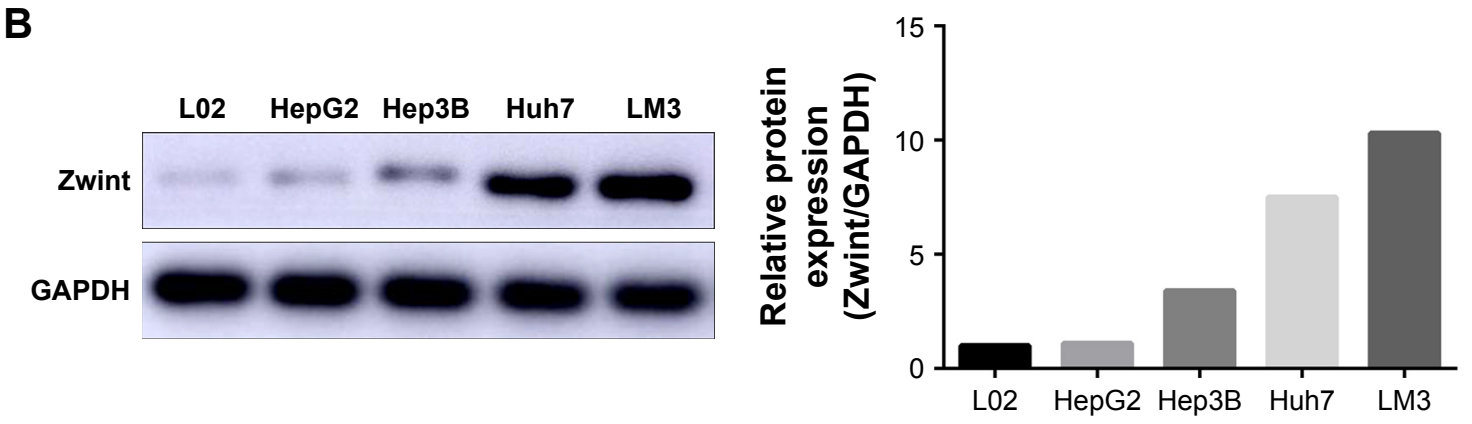

C
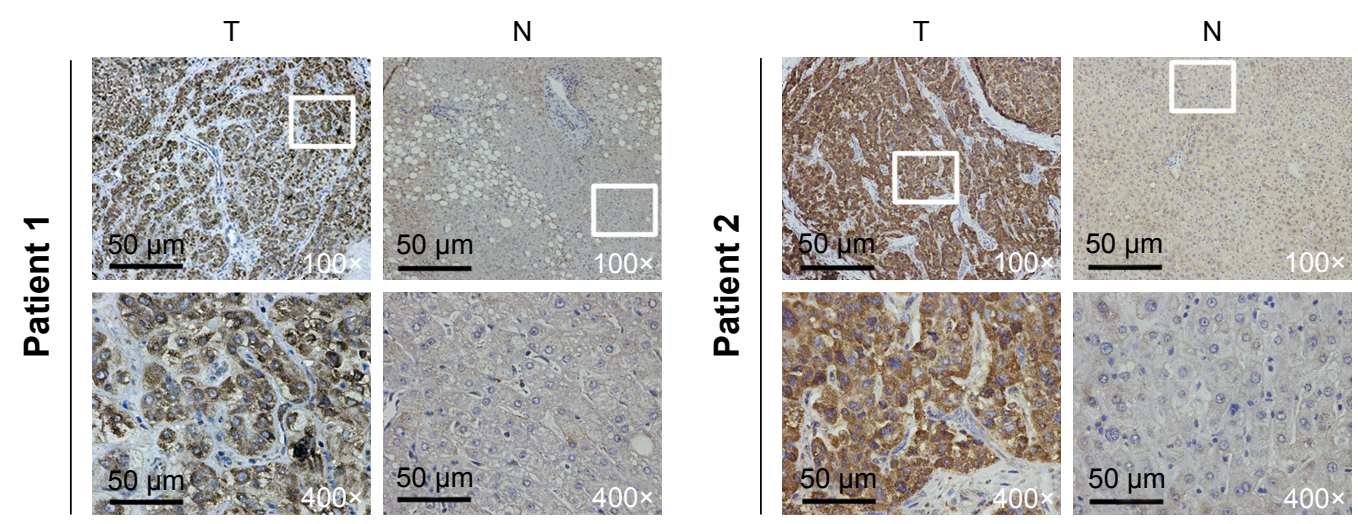

Figure 2 Expression of Zwint protein in HCC tissue and cell lines.

Notes: (A) Protein expression in $20 \mathrm{HCC}$ tissue samples and paired adjacent noncancerous tissue samples was determined by Western blot analysis. GAPDH was used as a loading control. (B) Protein expression of HCC cell lines and L02, a normal liver cell line. (C) Immunohistochemical staining of Zwint protein expression in HCC tissue and adjacent noncancerous tissue. The region in the white box is enlarged in the image in Figure 3.

Abbreviations: HCC, hepatocellular carcinoma; $\mathrm{T}$, tumor tissue; $\mathrm{N}$, noncancerous tissue.

$(P=0.005$, Figure $4 \mathrm{C})$ and disease-free survival $(P=0.008$, Figure 4D). Similarly, high expression of Zwint significantly predicted poor overall survival $(P=0.013$, Figure $4 \mathrm{E})$ and disease-free survival $(P=0.022$, Figure $4 \mathrm{~F})$ in patients with single tumors.

Cox regression analysis of evaluated independent risk factors was used, and univariate analysis showed that tumor size, tumor thrombi, TNM stage, and BCLC stage were significantly associated with overall and disease-free survival. Multivariate analysis showed that Zwint was an independent prognostic indicator of overall survival $(P=0.003)$ and disease-free survival $(P=0.022)$ (Table 2$)$.

\section{Zwint promoted HCC-cell proliferation}

Zwint upregulation was associated with tumor size and number, so Zwint may promote proliferation of HCC cells. shRNA knockdown of endogenous ZWINT in HCCLM3 and Huh7 cells was performed, and Zwint plasmid was used to express ZWINT mRNA and protein in HepG2 cells (Figure 5A and B). A CCK-8 assay (Figure 5C) showed that 

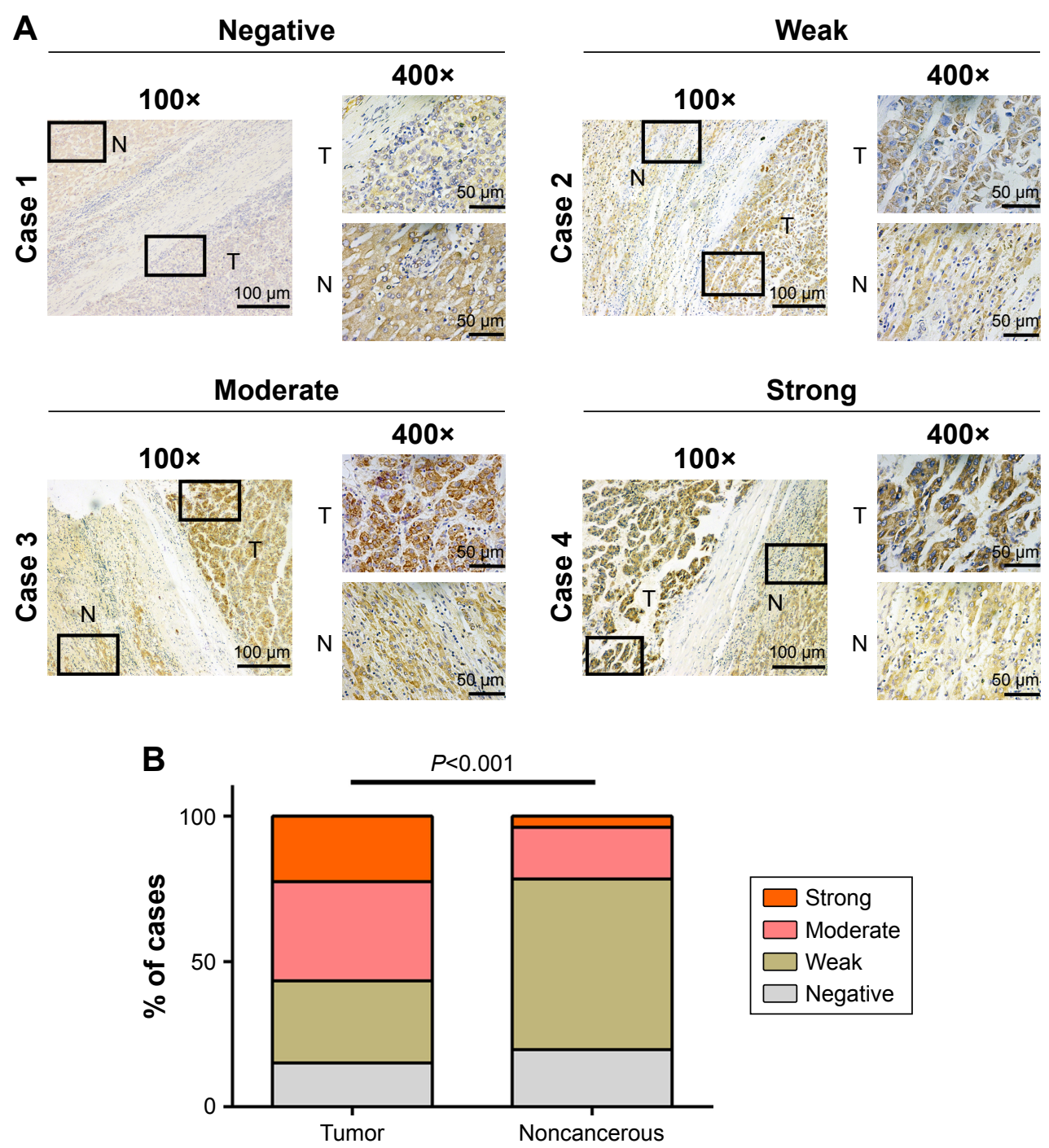

Figure 3 Immunostaining of Zwint protein in human HCC and adjacent normal tissues.

Notes: (A) Representative immunohistochemical expression patterns of Zwint in cancerous and adjacent normal tissue; (B) percentage of cases with different staining intensity of Zwint in the tumor or adjacent noncancerous tissue in the study cohort. $(n=\mid 06, P<0.00 I)$.

Abbreviations: HCC, hepatocellular carcinoma; T, tumor tissue; N, noncancerous tissue.

cell proliferation was significantly suppressed by ZWINT shRNA in HCCLM3 and Huh7 cells, and this was enhanced by ectopic expression of Zwint in HepG2 cells. Similarly, ZWINT knockdown attenuated clone formation for HCCLM3 and Huh7 cells, whereas ZWINT overexpression promoted clone formation in HepG2 cells (clones increased, Figure 5D).

\section{ZWINT knockdown arrested HCC cell cycle in $G_{2} / M$ phase}

Zwint protein's effect on the cell cycle was analyzed with flow cytometry after propidium iodide staining and Figure 6A shows that ZWINT knockdown significantly increased the proportion of HCCLM3 cells in the $\mathrm{G}_{2} / \mathrm{M}$ phase. Ectopic expression of Zwint decreased the proportion of HepG2 cells in the $\mathrm{G}_{2} / \mathrm{M}$ phase.

\section{ZWINT knockdown decreased the expression of cell-cycle proteins}

To elucidate the mechanism of cell-cycle arrest by ZWINT knockdown, we studied correlations between ZWINT mRNA expression and multiple genes with essential roles in the $\mathrm{G}_{2} / \mathrm{M}$ phase using the microarray data from 374 HCC tissues from the TCGA database. ZWINT expression was significantly correlated with $P C N A(P<0.001$, $R=0.691), C D C 25 C(P<0.001, R=0.814)$, cyclin B1 $(P<0.001, R=0.837)$, and $C D K 1$ expression $(P<0.001$, $R=0.909$ ) (Figure 6C). Furthermore, PCNA, cyclin B1, $\mathrm{Cdc} 25 \mathrm{C}$ and $\mathrm{CDK} 1$ protein quantified using Western blot (Figure 6B) showed that all were decreased in HCCLM3 cells after ZWINT knockdown and that these increased in HepG2 cells that overexpressed ZWINT. 

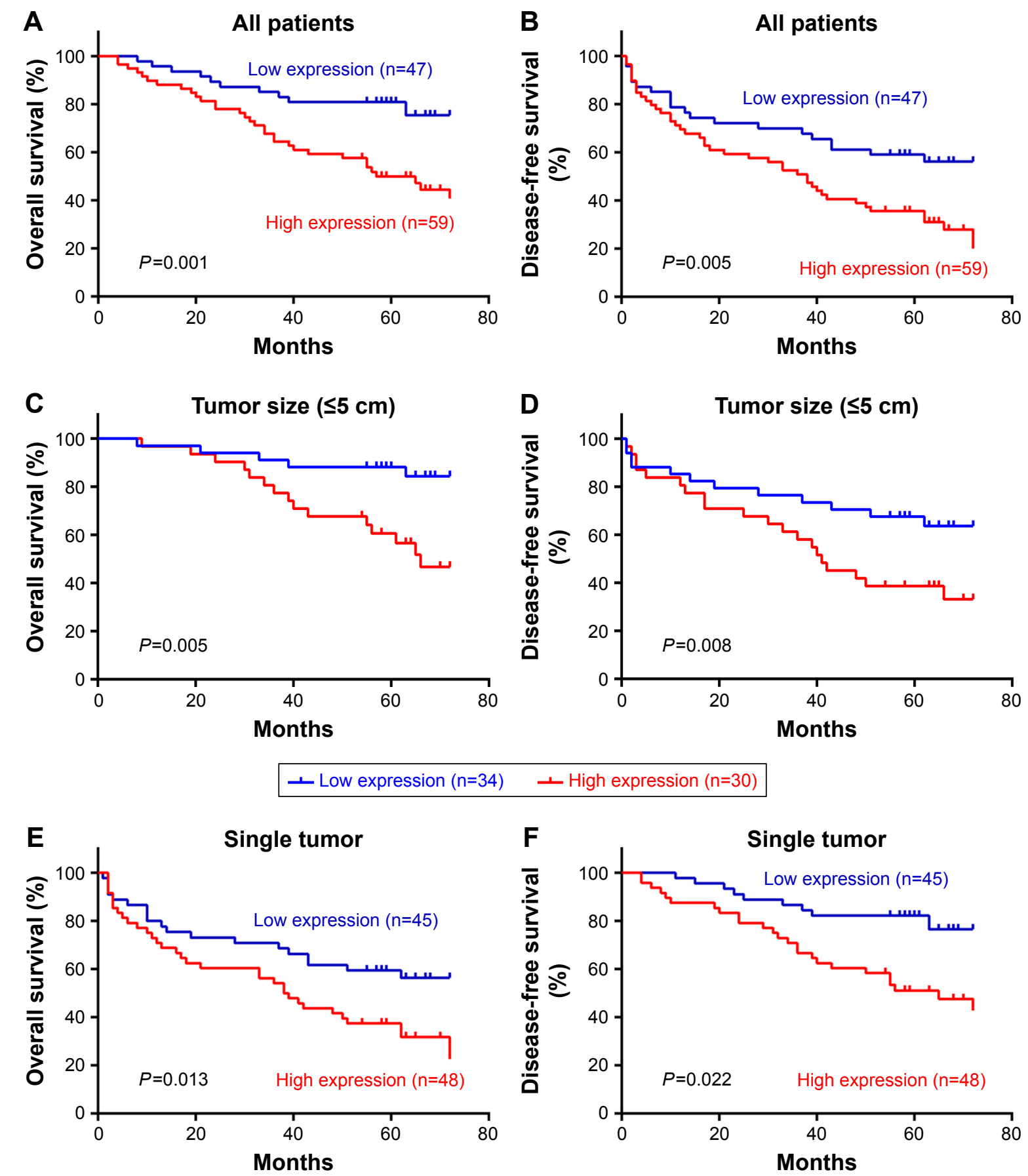

\section{+ High expression $(n=30)$}

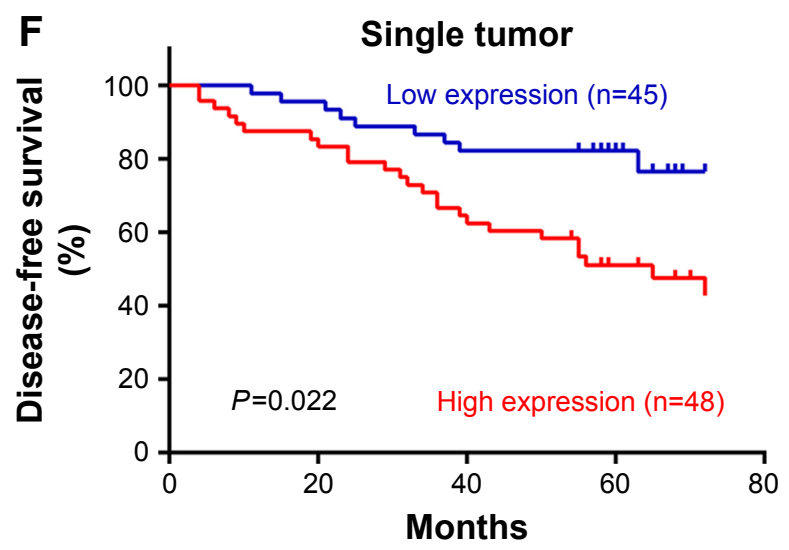

Figure 4 Kaplan-Meier survival analysis for HCC patients and subgroups.

Notes: (A, B) Kaplan-Meier curves for overall survival and disease-free survival of all HCC patients according to Zwint-expression status ( $\mathrm{n}=106$ ); (C, D) Kaplan-Meier curves for overall survival and disease-free survival of HCC patients with tumor size $\leq 5 \mathrm{~cm}$ according to Zwint-expression status ( $\mathrm{n}=64$ ); (E, F) Kaplan-Meier curves for overall survival and disease-free survival of HCC patients with single tumors according to Zwint-expression status ( $\mathrm{n}=93)$. $P$-values determined using log-rank tests.

Abbreviation: HCC, hepatocellular carcinoma.

\section{ZWINT knockdown inhibited tumor growth in vivo}

ZWINT knockdown decreased the proliferation of HCCLM3 cells in vitro, and this was confirmed in vivo using a stable HCCLM3 cell line for shZWINT with Scrambled shRNA used as a control. shZWINT decreased expression of Zwint and Ki67 compared with controls (Figure 7C-E). After the model had been established, tumors were completely removed 30 days later and imaged (Figure 7A). ZWINT knockdown decreased tumor volumes (Figure 7B), and qRT-PCR and Western blotting showed that ZWINT knockdown blocked expression of PCNA, cyclin B1, and CDK1 compared with controls. 
Table 2 Prognostic factors for OS and DFS of HCC patients $(n=106)$

\begin{tabular}{|c|c|c|c|c|c|c|c|c|}
\hline & \multicolumn{4}{|l|}{ OS } & \multicolumn{4}{|l|}{ DFS } \\
\hline & \multirow{2}{*}{$\frac{\text { Univariate }}{P \text {-value }}$} & \multicolumn{3}{|c|}{ Multivariate } & \multirow{2}{*}{$\frac{\text { Univariate }}{P \text {-value }}$} & \multicolumn{3}{|c|}{ Multivariate } \\
\hline & & $P$-value & HR & $95 \% \mathrm{Cl}$ & & $P$-value & HR & $95 \% \mathrm{Cl}$ \\
\hline Age (years), $<60 / \geq 60$ & 0.387 & & & & 0.118 & & & \\
\hline Sex & 0.727 & & & & 0.399 & & & \\
\hline HBsAg (negative/positive) & 0.577 & & & & 0.805 & & & \\
\hline $\operatorname{AFP}(\mathrm{ng} / \mathrm{mL}),<400 / \geq 400$ & 0.312 & & & & 0.984 & & & \\
\hline Liver cirrhosis (no/yes) & 0.78 & & & & 0.338 & & & \\
\hline Tumor size $(\mathrm{cm}),<5 / \geq 5$ & 0.009 & & & & 0.001 & 0.009 & 2.08 & $1.2-3.6$ \\
\hline Tumor number (single/multiple) & 0.705 & & & & 0.177 & & & \\
\hline Tumor differentiation $(I+I I / I I I+I V)$ & 0.367 & & & & 0.89 & & & \\
\hline Tumor thrombi (no/yes) & 0.049 & & & & $<0.001$ & 0.011 & 3.18 & $1.31-7.7$ \\
\hline TNM stage $(I+I I / I I I+I V)$ & 0.004 & & & & 0.004 & & & \\
\hline$B C L C$ stage $(A / B)$ & 0.002 & & & & $<0.001$ & & & \\
\hline Zwint (low/high) & 0.004 & 0.003 & 3.1 & $|.45-6.6|$ & 0.021 & 0.022 & 1.88 & I.I-3.22 \\
\hline
\end{tabular}

Abbreviations: OS, overall survival; DFS, disease-free survival; HCC, hepatocellular carcinoma; HBsAg, hepatitis B surface antigen; BCLC, Barcelona Clinic Liver Cancer; HR, hazard ratio.

\section{Discussion}

Zwint was initially identified as an additional centromereassociated protein in a yeast two-hybrid screen for proteins that interact with $\mathrm{Zw} 10$, regulating the mitotic spindle checkpoint. ${ }^{4}$ Knockdown of ZWINT disrupts localization of the RZZ complex (Rod, Zw10, and Zwilch) to centromeres and impairs checkpoint activity. ${ }^{5,21,22}$ Recently, Zwint was found to interact with the KMN network (Knl1, Mis12, and
A

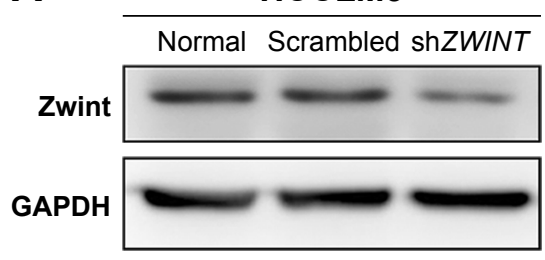

B

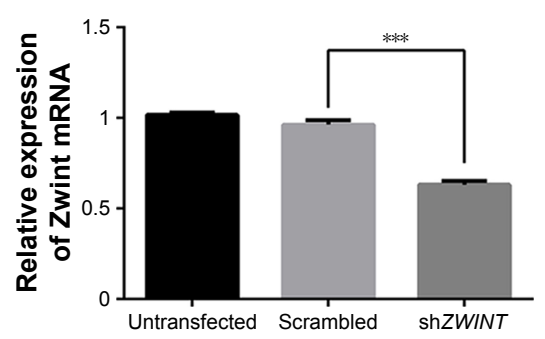

C

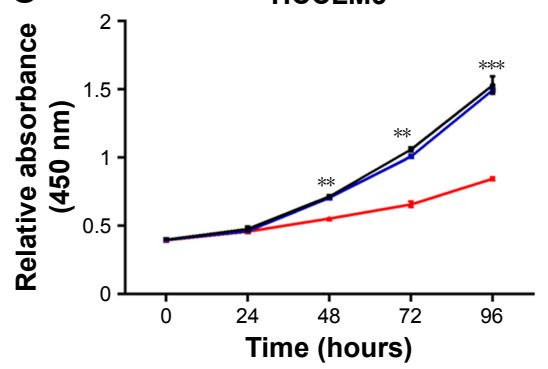

Huh7

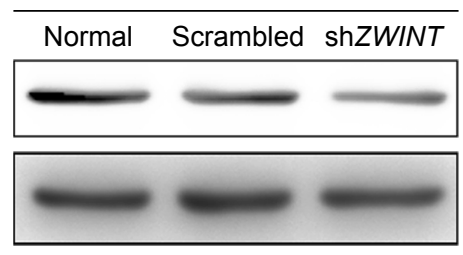

Huh7

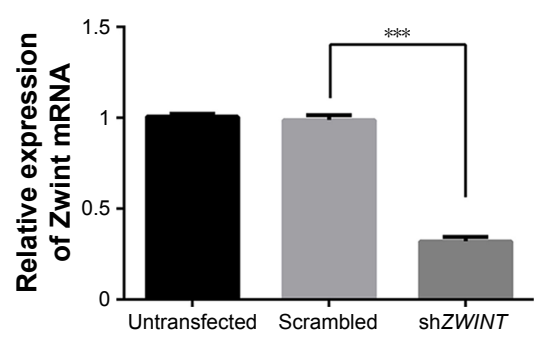

Huh7

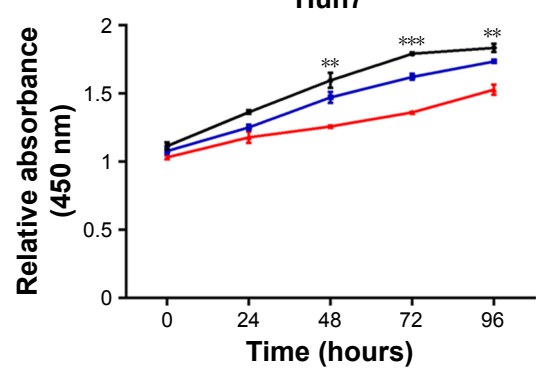

$\leftarrow$ shZWINT $\rightarrow$ Scrambled
HepG2

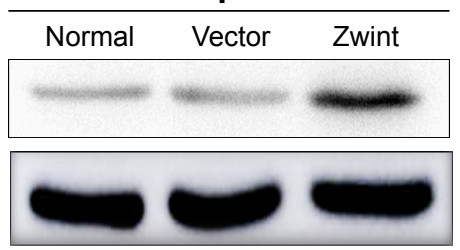

HepG2

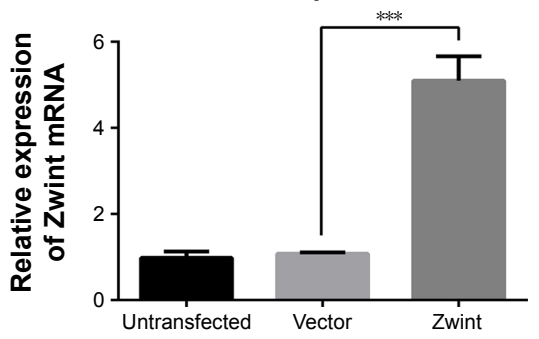

HepG2

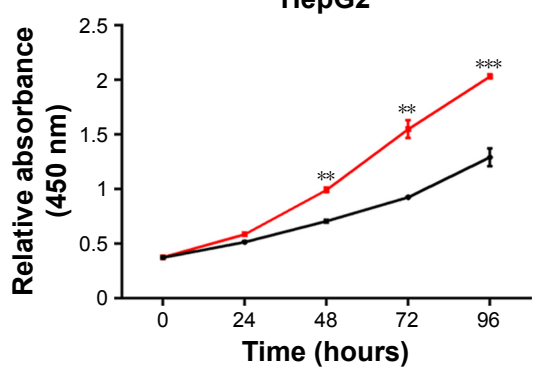

$\rightarrow$ Zwint $\rightarrow$ Vector

Figure 5 (Continued) 
D
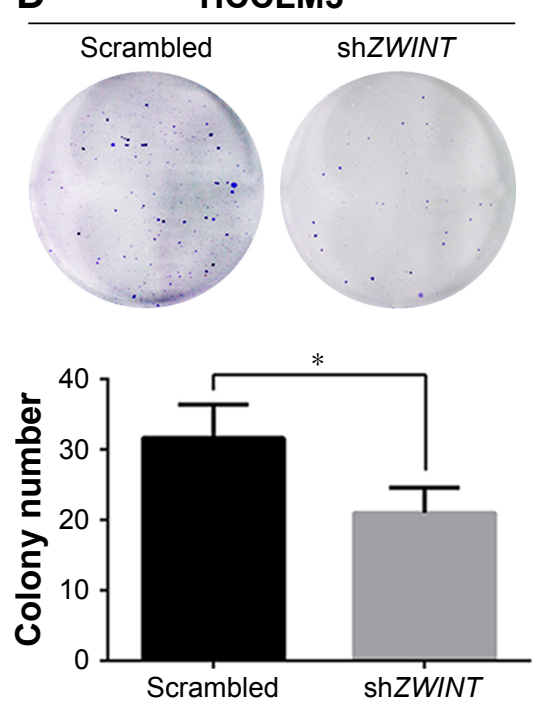

Huh7
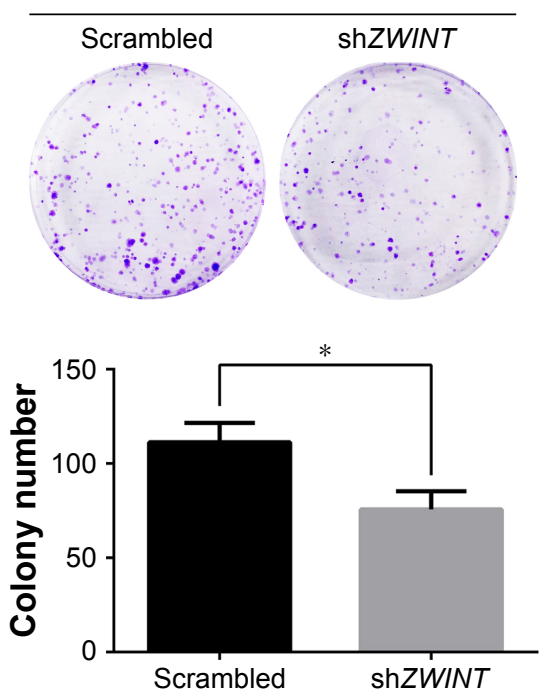

HepG2
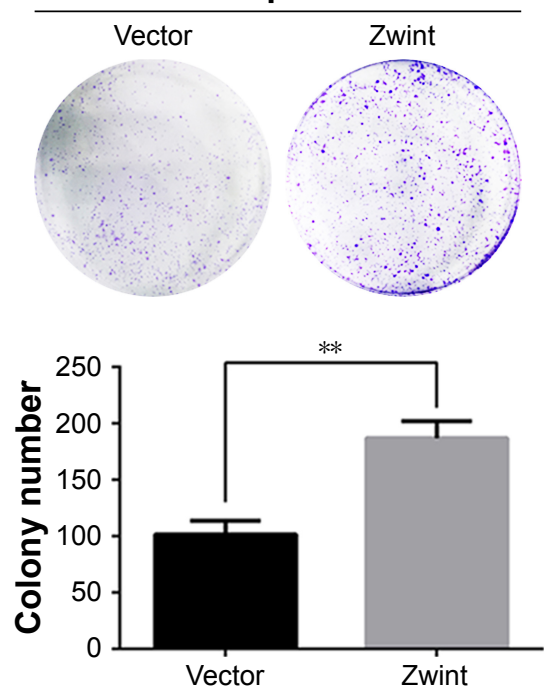

Figure 5 Effect of Zwint on proliferation of HCC cells.

Notes: (A) Zwint protein levels were detected in HCCLM3 and Huh7 cells with transfected shZWINT and HepG2 cells with transfected Zwint plasmid. (B) ZWINT mRNA expression detected in HCCLM3, Huh7, and HepG2 cells. (C) Cell growth of HCCLM3, Huh7, and HepG2 with shZWINT or Zwint plasmid determined by CCK-8 assay at different time points. (D) Knockdown of ZWINT suppressed cell growth of HCCLM3 and Huh7 in formation assays, and overexpressed ZWINT increased cell growth of HepG2. $* P<0.05 ; * * P<0.01 ; * * * p<0.001$.

Abbreviations: HCC, hepatocellular carcinoma; CCK-8, cell counting kit-8.

Ndc80) and appeared to connect the KMN network and the RZZ complex. This connection (KMN-Zwint-RZZ) is important for transferring signals from Aurora $\mathrm{B}$ to the spindle-assembly checkpoint. ${ }^{6,23-25}$ ZWINT is a gene that is overexpressed in pulmonary adenocarcinoma. ${ }^{11}$ Endoh et al reported that high expression of Zwint was related to poor survival in pulmonary adenocarcinoma. ${ }^{16}$ Brendle et al found that Zwint might affect breast cancer risk and clinical
A
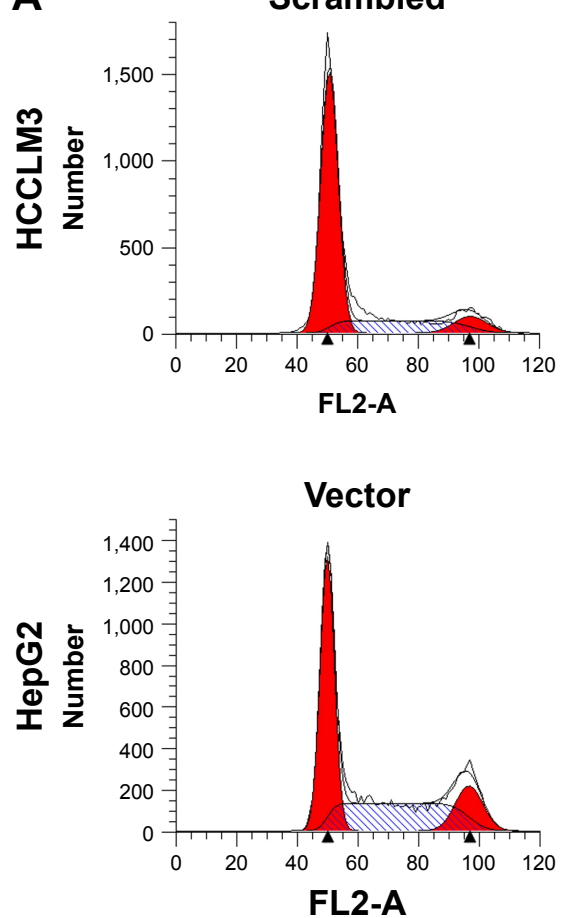

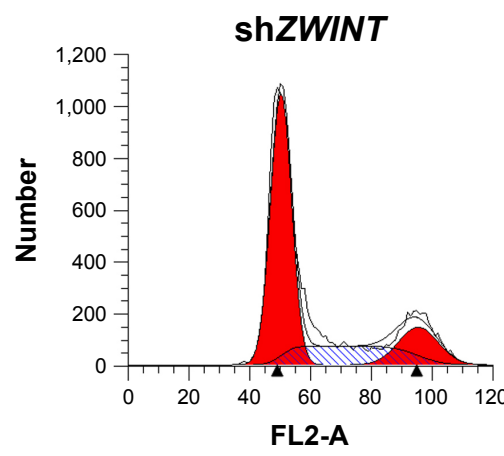

ZWINT

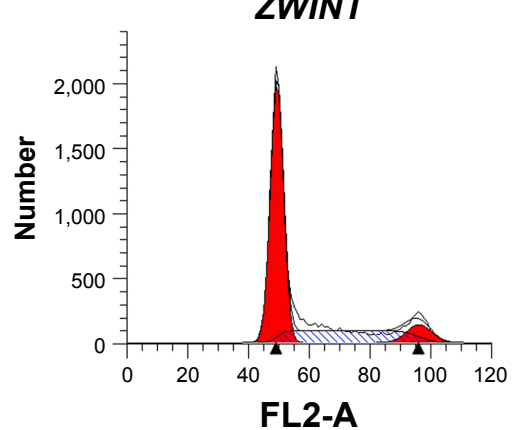

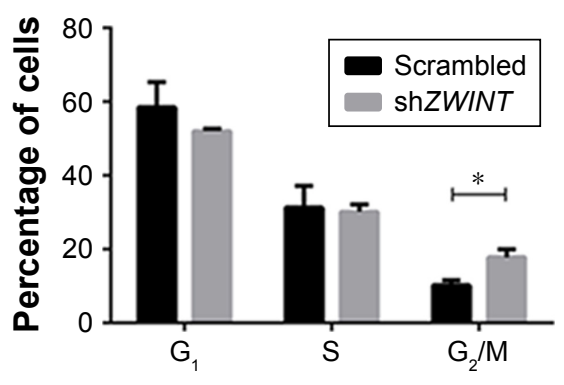

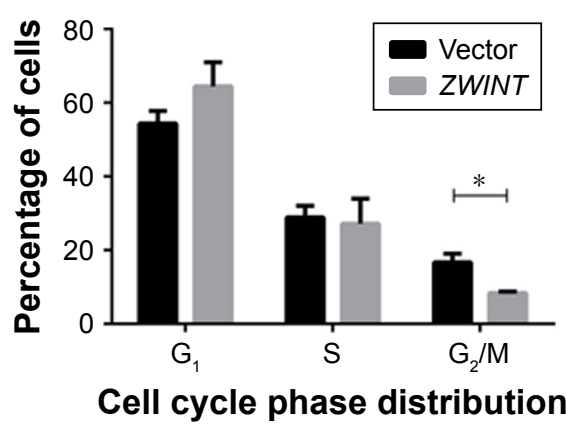

Figure 6 (Continued) 

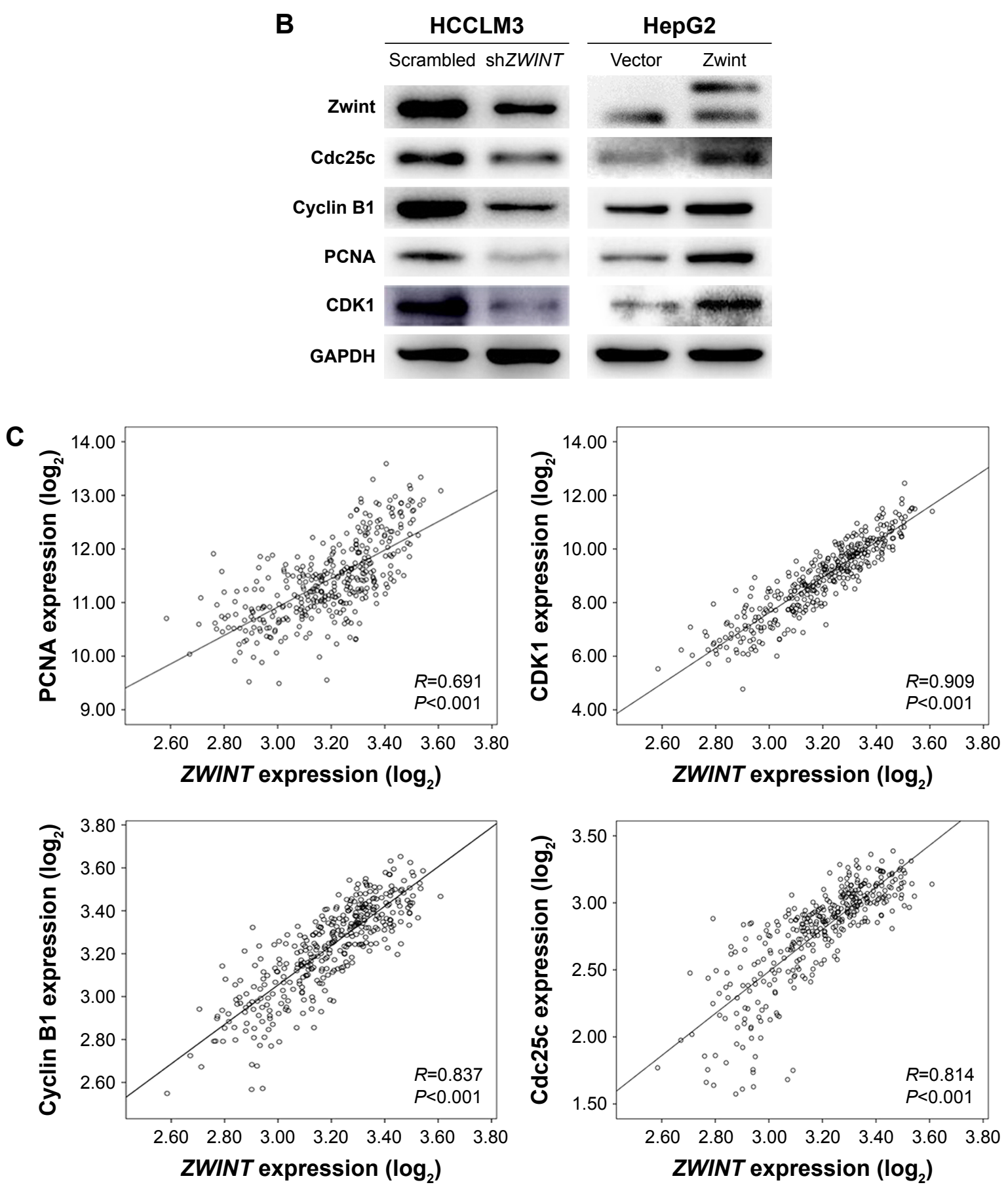

Figure 6 Effect of ZWINT on the cell cycle.

Notes: (A) Knocking down ZWINT induced $G_{2} / M$-phase arrest in HCCLM3, and upregulated ZWINT promoted the G/M phase in HepG2 cell lines. All fluorescenceactivated cell-sorting images representative of at least three independent experiments. The graphs at the right show quantification of the band intensity. (B) Protein levels of Zwint, PCNA, CDKI, cyclin BI, and Cdc25C in HCC cells with ZWINT knockdown. (C) Correlation between mRNA expression of ZWINT and PCNA, CDKI, cyclin BI and Cdc25C. mRNA expression of $374 \mathrm{HCC}$ patients from TCGA database. $* p<0.05$.

Abbreviations: HCC, hepatocellular carcinoma; TCGA, The Cancer Genome Atlas.

outcomes. ${ }^{26}$ Zwint could be upregulated by overexpression of androgen receptors in prostate cancer. ${ }^{15} \mathrm{Xu}$ et al recently demonstrated that ZWINT mRNA is frequently overexpressed in ovarian cancer, and may be a biomarker to support early diagnosis and evaluate prognosis. ${ }^{14}$ These results indicate that
Zwint may be significant in cancer and may be a potential target for cancer diagnosis and treatment. Even so, the correlation of Zwint and HCC has not been assessed.

We report that expression of Zwint is upregulated in $\mathrm{HCC}$ and may be used to predict tumor progression and 
A

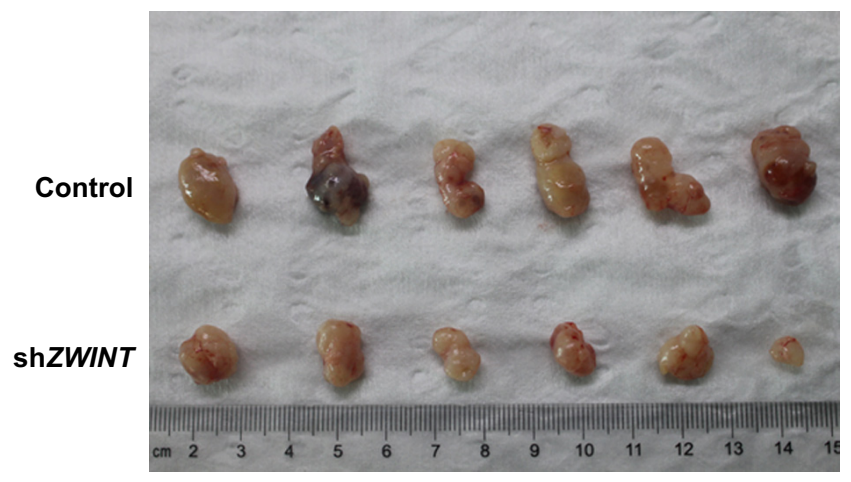

B

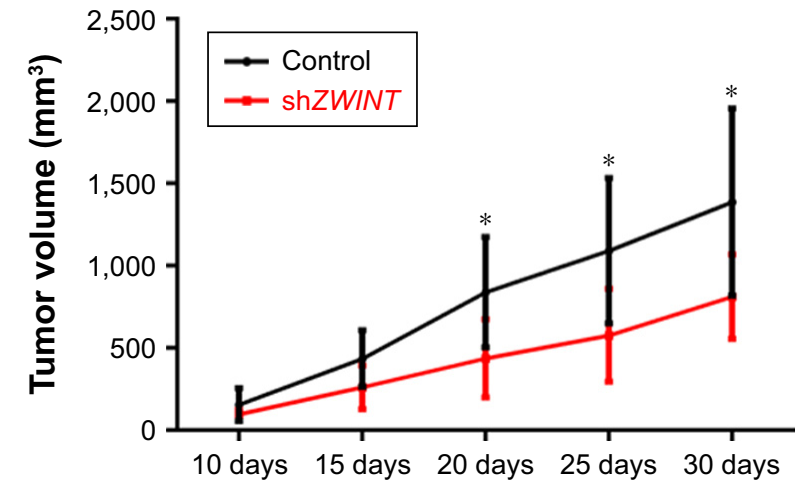

C Control shZWINT

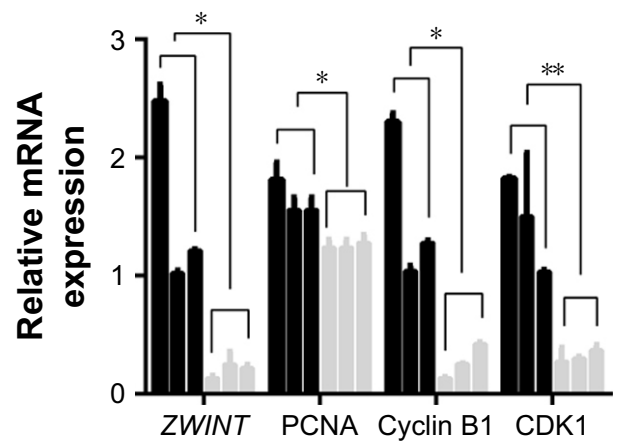

D

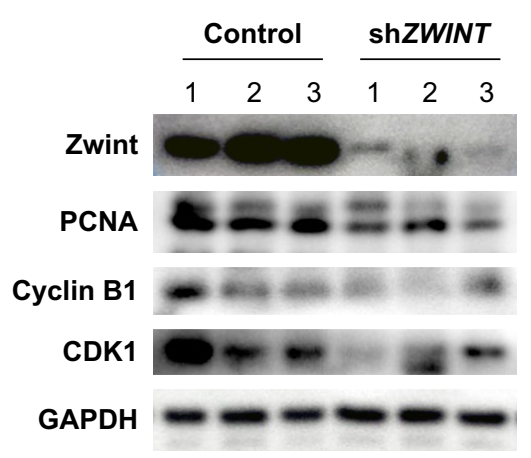

E

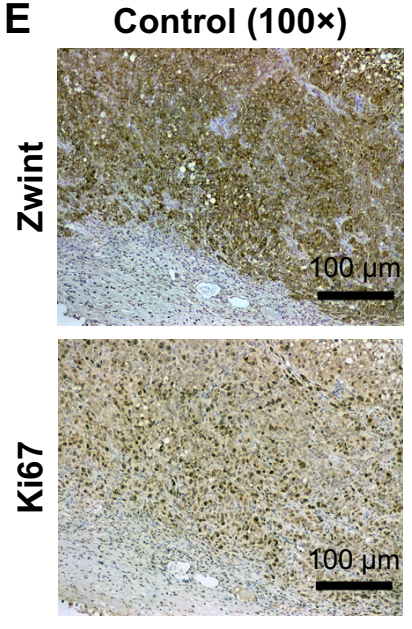

Control (200x)
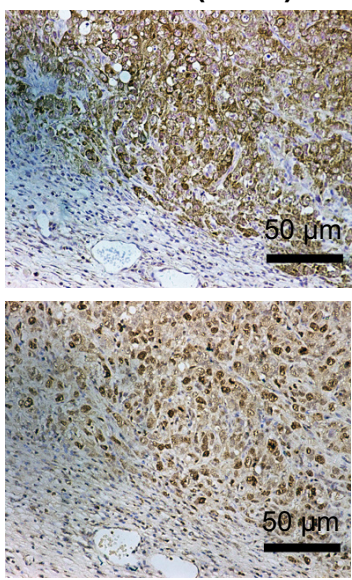

$\operatorname{shZWINT~(100x)~}$

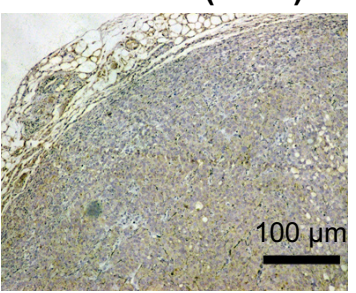

shZWINT (200x)
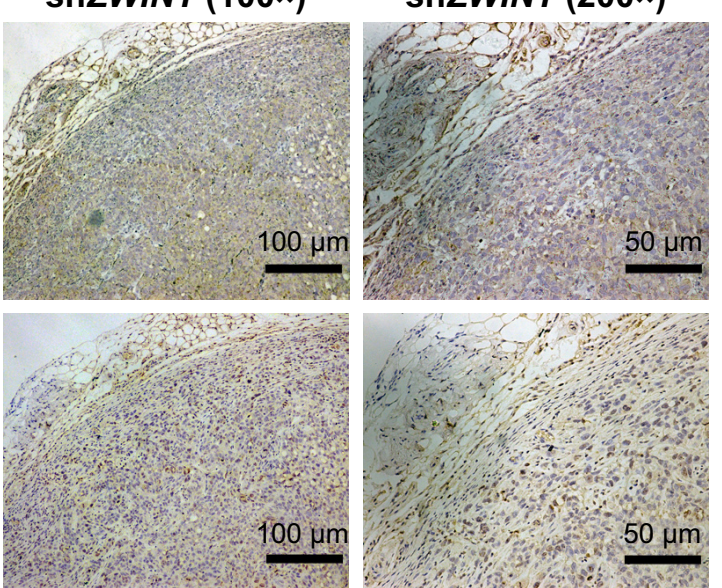

Figure 7 ZWINT knockdown inhibited tumor formation in vivo in nude mice.

Notes: (A) After 30 days, all nude mice were sacrificed and tumors separated. (B) Tumor volume calculated every 5 days ( $\mathrm{n}=6$ ). (C) mRNA expression of $Z$ WINT, PCNA, cyclin $\mathrm{BI}$ and CDKI in tumor tissue of control and shZWINT groups (means \pm standard error of the mean). (D) Western blot shows protein expression of Zwint, PCNA, cyclin $\mathrm{BI}$, and CDKI. (E) Immunohistochemistry images of Zwint and Ki67 expression in control and shZWINT groups. $* P<0.05 ; * * P<0.01$.

Abbreviation: HCC, hepatocellular carcinoma.

unfavorable prognosis of HCC patients. First, we collected information about Zwint expression from the Oncomine database. Two independent data sets showed that ZWINT mRNA was increased in HCC tissue compared with normal liver tissue. Data from a group of 364 HCC patients with information about mRNA expression and prognosis was obtained from the TCGA database using bioinformatic analysis, and high expression of Zwint predicted a poor rate of survival. Then, with qRT-PCR and Western blot analysis, we found that Zwint was overexpressed in HCC samples and HCC cell lines. Data support previous findings about upregulated ZWINT mRNA expression in human cancers.

Furthermore, our IHC analysis of 106 paired HCC samples confirmed that the Zwint protein was significantly 
upregulated in HCC; $55.6 \%$ of cancerous tissue had moderate or high expression, whereas only $21.7 \%$ of adjacent noncancerous tissue had similar results with the IHC. Of note, significant differences were observed with qRT-PCR $(86.5 \%)$ and Western blotting (90\%) in cancerous tissues. This discrepancy may have been related to the number of HCC samples. Therefore, our results confirmed that Zwint is significantly upregulated in HCC.

A more important finding of our study was a correlation of clinicopathologic features and the prognostic value of Zwint expression in HCC. High expression of Zwint was significantly correlated with tumor size and number, indicating that Zwint may be a biomarker to evaluate the progression of HCC patients. In addition, we observed a significant association between increased Zwint protein and poor survival and high recurrence of HCC. Univariate and multivariate survival analysis showed that increased Zwint expression was significantly associated with overall and disease-free survival, and was an independent risk factor for poor survival and high recurrence in HCC patients. Tumor characteristics, such as tumor number and size, are important for progression and prognosis of $\mathrm{HCC}$, and patients at the same clinical stage may have different outcomes. ${ }^{27}$ Therefore, we performed a stratified survival analysis, and demonstrated that high expression of Zwint also predicted poor overall survival and disease-free survival for patients with $\leq 5 \mathrm{~cm}$ tumors and single tumors. Therefore, results from our study suggest that Zwint expression may be a biomarker to predict prognosis in HCC patients. This may also be used to classify patients into distinct risk subgroups, as well as serve as a novel therapeutic target of HCC.

It has been documented that Zwint is required for spindleassembly checkpoint function. Downregulation of Zwint may induce Zw10 centromere instability and negatively regulate cell proliferation in $293 \mathrm{~T}$ and breast cancer cells. To analyze the biological function of Zwint, we knocked down or overexpressed ZWINT in HCC cells. Proliferation, clone formation, and the cell cycle were reduced with shZWINT, whereas these functions increased in HCC cells over-expressing ZWINT. Cell-cycle studies in HCC cells confirmed that there were more cells in the $G_{2} / M$ phase, whereas cells in the $G_{1}$ or $S$ phase were reduced. Multiple genes that regulate the $G_{2} / M$ phase were assayed using mRNA-expression data from 374 HCC samples from the TCGA database. Zwint expression was significantly correlated with PCNA, Cdc25C, cyclin B1, and CDK1 expression. PCNA, Cdc25C, cyclin B1, and $\mathrm{CDK} 1$ proteins were also positively regulated in $\mathrm{HCC}$ cell lines that were studied, with downregulated or upregulated
Zwint expression. Therefore, expression of Zwint might contribute to the HCC cell cycle by regulating the $\mathrm{G}_{2} / \mathrm{M}$ phase. However, how Zwint and cell-cycle proteins interact is not clear, and more studies are required.

In conclusion, we showed that expression of Zwint was frequently upregulated in HCC tissues and HCC cell lines. High expression of Zwint was significantly associated with tumor progression and poor prognosis of HCC patients. In HCC cell lines, depletion of Zwint may attenuate proliferation and cell-cycle progression of HCC cells, but our study was limited in many ways. We did find a correlation between Zwint and cell-cycle proteins, but how Zwint affects proliferation and the cell cycle is not clear. As such, Zwint might be a novel biomarker for HCC progression and prognosis, but more studies are required to validate this preliminary data and clarify a role for Zwint in HCC.

\section{Acknowledgments}

We acknowledge Hui Liu and Qi Wang for statistical analysis and checking the text thoroughly for accuracy. We would like to thank LetPub (www.letpub.com) for providing linguistic assistance during the preparation of this manuscript.

\section{Disclosure}

The authors report no conflicts of interest in this work.

\section{References}

1. Torre LA, Bray F, Siegel RL, Ferlay J, Lortet-Tieulent J, Jemal A. Global cancer statistics, 2012. CA Cancer J Clin. 2015;65(2):87-108.

2. Motola-Kuba D, Zamora-Valdés D, Uribe M, Méndez-Sánchez N Hepatocellular carcinoma: an overview. Ann Hepatol. 2006;5(1):16-24.

3. Pan HW, Ou YH, Peng SY, et al. Overexpression of osteopontin is associated with intrahepatic metastasis, early recurrence, and poorer prognosis of surgically resected hepatocellular carcinoma. Cancer 2003;98(1):119-127.

4. Starr DA, Saffery R, Li Z, et al. HZwint-1, a novel human kinetochore component that interacts with HZW10. J Cell Sci. 2000;113(Pt 11): 1939-1950.

5. Famulski JK, Vos L, Sun X, Chan G. Stable hZW10 kinetochore residency, mediated by hZwint-1 interaction, is essential for the mitotic checkpoint. J Cell Biol. 2008;180(3):507-520.

6. Obuse C, Iwasaki O, Kiyomitsu T, Goshima G, Toyoda Y, Yanagida M. A conserved Mis12 centromere complex is linked to heterochromatic HP1 and outer kinetochore protein Zwint-1. Nat Cell Biol. 2004; 6(11):1135-1141.

7. Cheeseman IM, Niessen S, Anderson S, et al. A conserved protein network controls assembly of the outer kinetochore and its ability to sustain tension. Genes Dev. 2004;18(18):2255-2268.

8. van Vlijmen T, Vleugel $M$, Evers $M$, et al. A unique residue in rab3c determines the interaction with novel binding protein Zwint-1 FEBS Lett. 2008;582(19):2838-2842.

9. Endo H, Ikeda K, Urano T, Horie-Inoue K, Inoue S. Terf/TRIM17 stimulates degradation of kinetochore protein ZWINT and regulates cell proliferation. J Biochem. 2012;151(2):139-144.

10. Seo DW, You SY, Chung WJ, Cho DH, Kim JS, Oh JS. Zwint-1 is required for spindle assembly checkpoint function and kinetochoremicrotubule attachment during oocyte meiosis. Sci Rep. 2015;5:15431. 
11. Bhattacharjee A, Richards WG, Staunton J, et al. Classification of human lung carcinomas by mRNA expression profiling reveals distinct adenocarcinoma subclasses. Proc Natl Acad Sci U S A. 2001;98(24): 13790-13795.

12. Ho JR, Chapeaublanc E, Kirkwood L, et al. Deregulation of Rab and Rab effector genes in bladder cancer. PLOS One. 2012;7(6):e39469.

13. Pérez-Ramírez M, Hernández-Jiménez AJ, Guerrero-Guerrero A, et al. Genomics and epigenetics: a study of ependymomas in pediatric patients. Clin Neurol Neurosurg. 2016;144:53-58.

14. Xu Z, Zhou Y, Cao Y, Dinh TL, Wan J, Zhao M. Identification of candidate biomarkers and analysis of prognostic values in ovarian cancer by integrated bioinformatics analysis. Med Oncol. 2016;33(11):130.

15. Urbanucci A, Sahu B, Seppälä J, et al. Overexpression of androgen receptor enhances the binding of the receptor to the chromatin in prostate cancer. Oncogene. 2012;31(17):2153-2163.

16. Endoh H, Tomida S, Yatabe Y, et al. Prognostic model of pulmonary adenocarcinoma by expression profiling of eight genes as determined by quantitative real-time reverse transcriptase polymerase chain reaction. J Clin Oncol. 2004;22(5):811-819.

17. de Castro IP, de Cárcer G, Malumbres M. A census of mitotic cancer genes: new insights into tumor cell biology and cancer therapy. Carcinogenesis. 2007;28(5):899-912.

18. Edge SB, Compton CC. The American Joint Committee on Cancer: the 7 th edition of the AJCC Cancer Staging Manual and the future of TNM. Ann Surg Oncol. 2010;17(6):1471-1474.

19. Llovet JM, Di Bisceglie AM, Bruix J, et al. Design and endpoints of clinical trials in hepatocellular carcinoma. J Natl Cancer Inst. 2008; 100(10):698-711.
20. Tang L, Tan YX, Jiang BG, et al. The prognostic significance and therapeutic potential of hedgehog signaling in intrahepatic cholangiocellular carcinoma. Clin Cancer Res. 2013;19(8):2014-2024.

21. Wang H, Hu X, Ding X, et al. Human Zwint-1 specifies localization of Zeste White 10 to kinetochores and is essential for mitotic checkpoint signaling. J Biol Chem. 2004;279(52):54590-54598.

22. Kops GJ, Kim Y, Weaver BA, et al. ZW10 links mitotic checkpoint signaling to the structural kinetochore. J Cell Biol. 2005;169(1):49-60.

23. Lin YT, Chen Y, Wu G, Lee WH. Hec1 sequentially recruits Zwint-1 and ZW10 to kinetochores for faithful chromosome segregation and spindle checkpoint control. Oncogene. 2006;25(52):6901-6914.

24. Vos LJ, Famulski JK, Chan GK. hZwint-1 bridges the inner and outer kinetochore: identification of the kinetochore localization domain and the hZw10-interaction domain. Biochem J. 2011;436(1):157-168.

25. Kasuboski JM, Bader JR, Vaughan PS, et al. Zwint-1 is a novel Aurora B substrate required for the assembly of a dynein-binding platform on kinetochores. Mol Biol Cell. 2011;22(18):3318-3330.

26. Brendle A, Brandt A, Johansson R, et al. Single nucleotide polymorphisms in chromosomal instability genes and risk and clinical outcome of breast cancer: a Swedish prospective case-control study. Eur J Cancer. 2009;45(3):435-442.

27. Lee JS, Heo J, Libbrecht L, et al. A novel prognostic subtype of human hepatocellular carcinoma derived from hepatic progenitor cells. Nat Med. 2006;12(4):410-416.

28. Tang Z, Li C, Kang B, et al. GEPIA: a web server for cancer and normal gene expression profiling and interactive analyses. Nucleic Acids Res. 2017;45: W98-W102.
OncoTargets and Therapy

\section{Publish your work in this journal}

OncoTargets and Therapy is an international, peer-reviewed, open access journal focusing on the pathological basis of all cancers, potential targets for therapy and treatment protocols employed to improve the management of cancer patients. The journal also focuses on the impact of management programs and new therapeutic agents and protocols on

\section{Dovepress}

patient perspectives such as quality of life, adherence and satisfaction The manuscript management system is completely online and includes a very quick and fair peer-review system, which is all easy to use. Visit http://www.dovepress.com/testimonials.php to read real quotes from published authors. 